\title{
Modelado 3D CAD de una Planta Potabilizadora
}

\author{
Caballero, Rodrigo \\ Facultad de Ingeniería Mecánica, Universidad Tecnológica de Panamá \\ Panamá, Panamá \\ rodrigo.caballero@utp.ac.pa \\ Castrellón, María \\ Universidad del Istmo \\ Panamá, Panamá \\ modelacion.matematica@udelistmo.edu \\ Pulido, Gonzalo \\ Universidad del Istmo \\ Panamá, Panamá \\ gpulido@udelistmo.edu
}

\section{Abstract}

This article shows the usefulness of developing three-dimensional (3D) models using Computer Aided Design (CAD) techniques as input data for the mathematical modelling of the flow fields in various unit operations of a water treatment plant, namely: rapid mixing, pre-flocculation, flocculation, sedimentation, filtration and contact tank. The 3D CAD models being developed for this purpose input the geometrical information of the unit operations as they exist in the water treatment plant today. After obtaining results from the mathematical modelling, modifications to various units will be proposed, which will require new versions of the 3D CAD models to be input into the mathematical model at the flow prediction stage under plant design modifications. CAD techniques are also being used in 2D to make asbuilt drawings of the entire water treatment plant. The software used is AutoCAD $尺$ and AutoCAD civil 3D®.

Keywords: Computer Aided Design, mathematical modeling, 3D modelling, Computacional Fluid Dynamics, virtual. 


\section{Resumen}

En este artículo se muestra la utilidad de desarrollar modelos tridimensionales (3D) usando técnicas de Diseño Asistido por Computador (CAD, por sus siglas en Ingles), como datos de entrada para la modelación matemática de los campos de flujo en diversas operaciones unitarias de una planta potabilizadora, a saber: mezcla rápida, prefloculación, floculación, sedimentación, filtración y tanque de contacto. Los modelos CAD 3D que se están desarrollando para este propósito, ingresan la información geométrica de las operaciones unitarias tal y como existen en la potabilizadora en la actualidad. Después de obtener resultados de la modelación matemática, se propondrán modificaciones a varias unidades, lo que requerirá nuevas versiones de los modelos CAD 3D, para ser ingresados al modelo matemático en fase de predicción de flujo bajo modificaciones de diseño en la planta. Las técnicas CAD también se están usando en 2D para hacer los planos "como construidos" de toda la potabilizadora. El software utilizado es AutoCAD ${ }^{\circledR}$ y AutoCAD civil 3D ${ }^{\circledR}$.

Palabras claves: Diseño Asistido por Computador, modelación matemática, modelado 3D, Dinámica de Fluido Computacional, virtual.

\section{INTRODUCCIÓN}

La importancia de una óptima documentación técnica a llegado a los niveles de tener que definir normas que establezcan su claridad, efectividad y precisión con el objetivo de poder crear, diseñar y modificar; es aquí donde los sistemas "Diseño asistido por computadora (CAD)", juegan un rol cada día más importante ya que este tipo de software hoy en día no solo permiten la realización de planos habituales que luego puedan ser editados, si no que nos permite ejecutar sistemas tridimensionales los cuales hoy día son cruciales para la preparación operativa para mejorar las compilaciones de datos de activos para las plantas, la importancia del modelado CAD 3D se hace evidente a medida que la industria se vuelve más digital. [1] Con el aumento de la competencia internacional, el aumento de los costos y la fluctuación de los precios de la energía, hay un gran énfasis en mejorar la rentabilidad con proyectos más eficientes y tiempos de respuesta más cortos. Las implicaciones que poseen la modelación 3D en la actualidad aumentan significativamente en las áreas medicinales, civiles, fabricación, análisis estructurales por método de elemento finito, simulaciones de líneas de flujo de transición y estudios por medio de simulaciones por modelos matemáticos establecidos, tal como bien se implementa en el proyecto "Formulación de la Optimización de una Potabilizadora por Modelación Matemática e Instrumentación In-Situ". Con el 
objetivo de alcanzar en esta fase del proyecto:

1. Establecer sistemas de documentación digital que permitan establecer mediciones y registros en la potabilizadora en base de algoritmo simplificados y concretos.

2. Reconocer y aplicar los conceptos analíticos que modelan matemáticamente diferentes situaciones de la vida real o del área académica para dar una interpretación válida en el contexto del problema, en base a datos previos prexistentes.

3. Desarrollar e implementar simulaciones de modelaciones fisicoquímicas y matemáticas, para el análisis y optimización por medio de modelación 3D CAD y CFD.

4. Crear de un modelo CAD 3D para uso como dato de entrada para OpenFOAM.

\section{MÉTODO}

El proceso de desarrollo de un modelado 3D en CAD que se está desarrollando en la potabilizadora Jaime Diaz Quintero (JDQ) implica un conjunto de técnicas que van desde los registros tradicionales tales como recolección de información, análisis de mediciones topográficas, así como la implementación de técnicas modernas ingenieriles de elaboraciones de entornos ajustados a sistemas reales.

INFORMACIÓN: Compilación de la mayor cantidad de documentaciones técnicas prexistentes tales como planos estructurales, fotografías de proyectos, así como registros generales de construcciones y sistemas instalados.

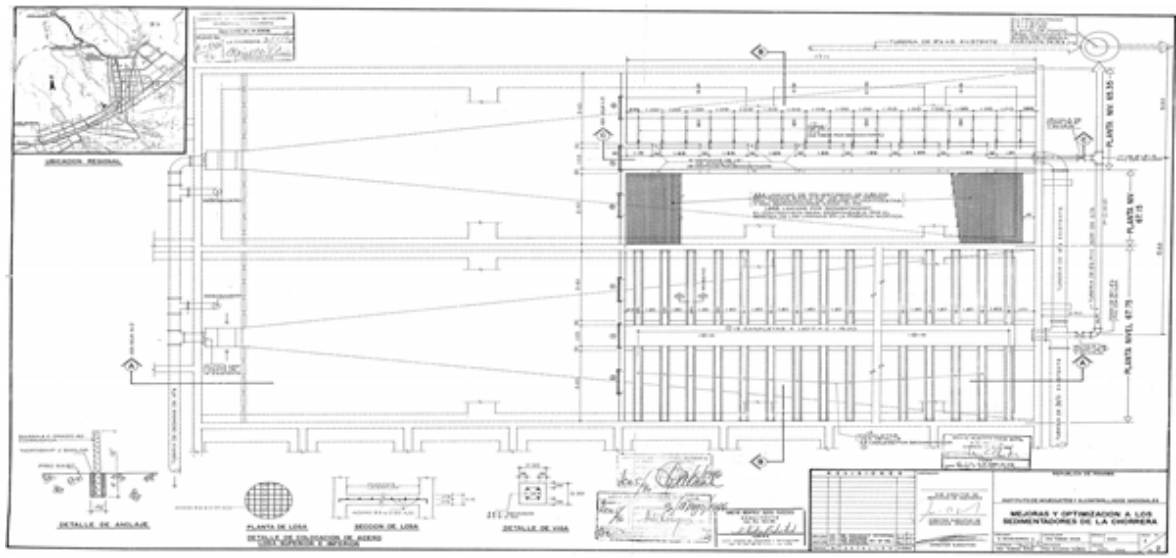

Fig. 1 Plano de mejoras y optimización en planta potabilizadora en 1996.

REGISTRO Y RECTIFICACIÓN: Captación de datos para la modelación 3D, por lo cual se emplea el uso de una estación total Leica FlexLine TS06, para el levantamiento mediciones topográficas, estructurales y de nuevas modificaciones, así como la verificación de los datos existente. Además, se procedió con una investigación de equipos y piezas instaladas con datos de fabricantes. 

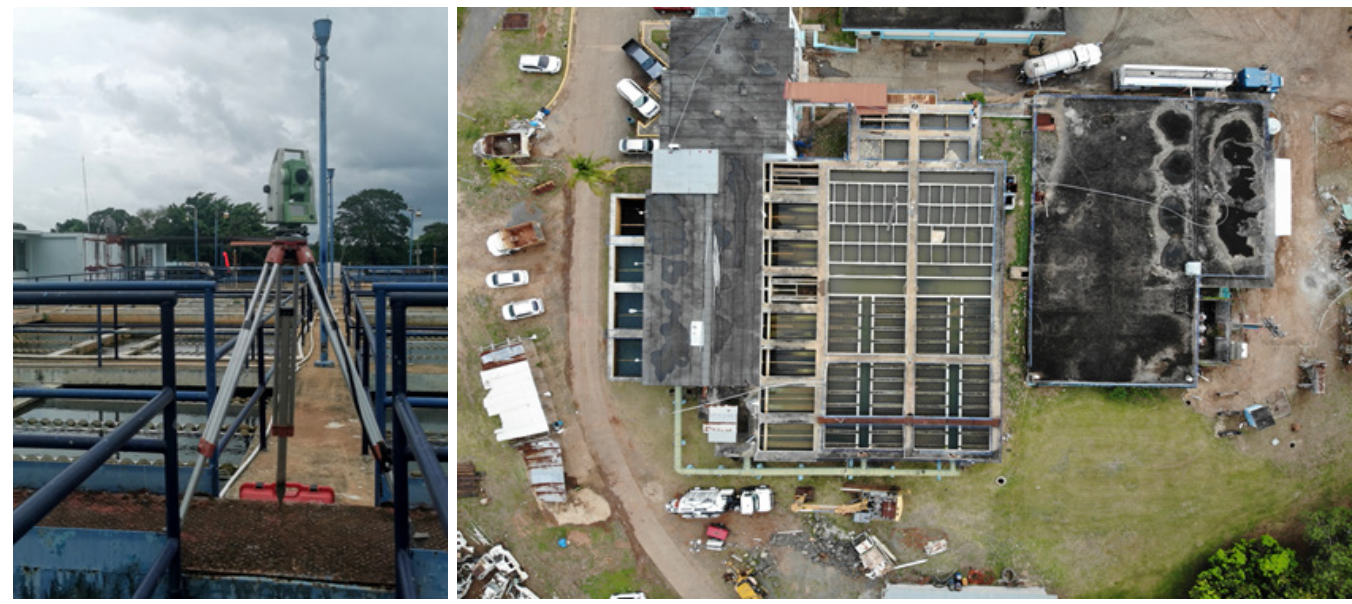

Fig. 2 Medición y rectificación In-Situ. a) Estación total marca Leica FlexLine TS06; b) Imagen aérea planta potabilizadora de la Chorrera

MODELADO 3D: El modelo 3D por medio se softwares CAD implementado incluye datos y características reales de las unidades y sistemas presentes que integran la potabilizara, estableciendo una herramienta que permite elaborar planos estructurales tradicionales y futuras modificaciones en planta. Para ello se emplea el AutoCAD civil 3D que por medio de su sistema de algoritmo transforma los datos topográficos registrado por la estación total en un sistema vectorial a un modelo real tridimensional de las áreas superficiales. [2]

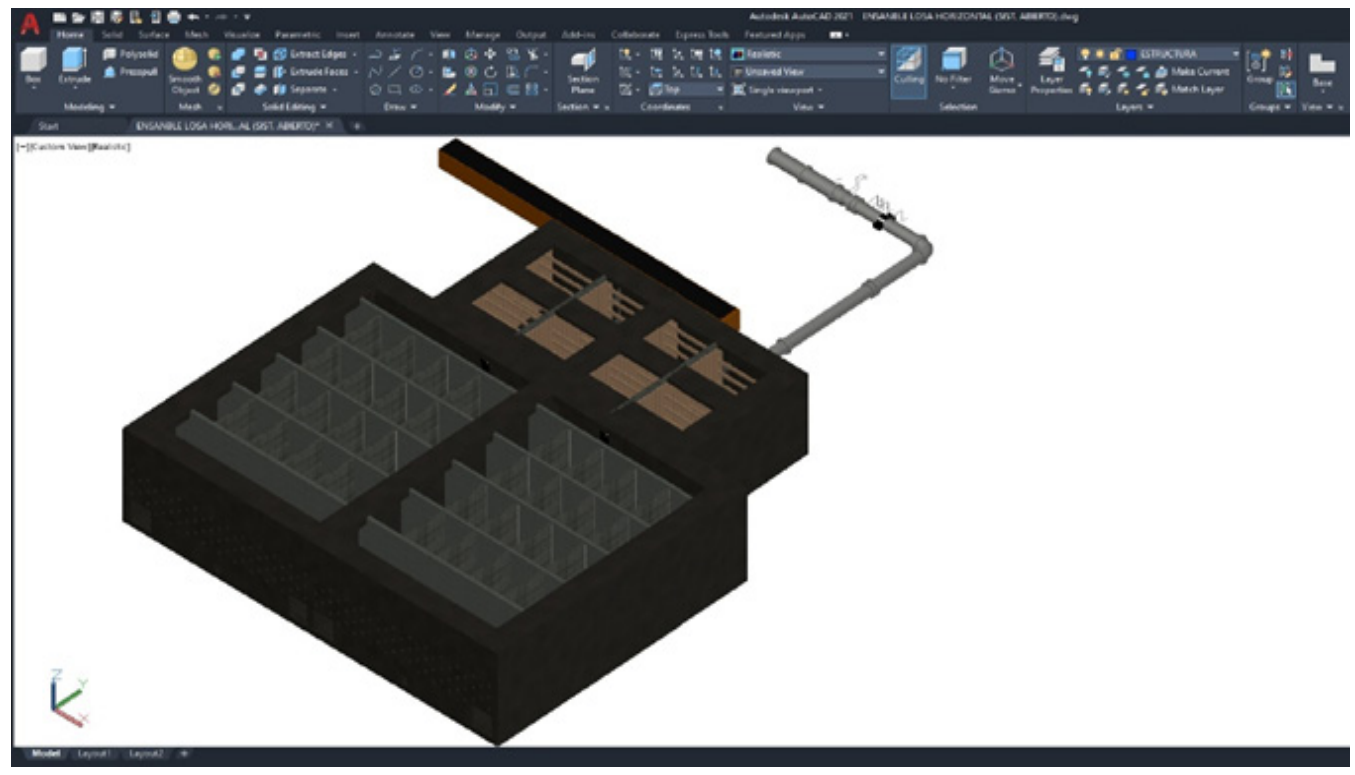

Fig. 3 Modelado 3D realizado en AutoCAD (Pre-floculadores, floculadores y mezcla rápida).

IMPLEMENTACIÓN: La modelación tridimensional en AutoCAD elaborada se empleará como base de la modelación matemática de las diferentes operaciones unitarias de la potabilizadora con técnicas de Dinámica de Fluido Computacional (CFD) específicamente 
usando la plataforma OpenFOAM.

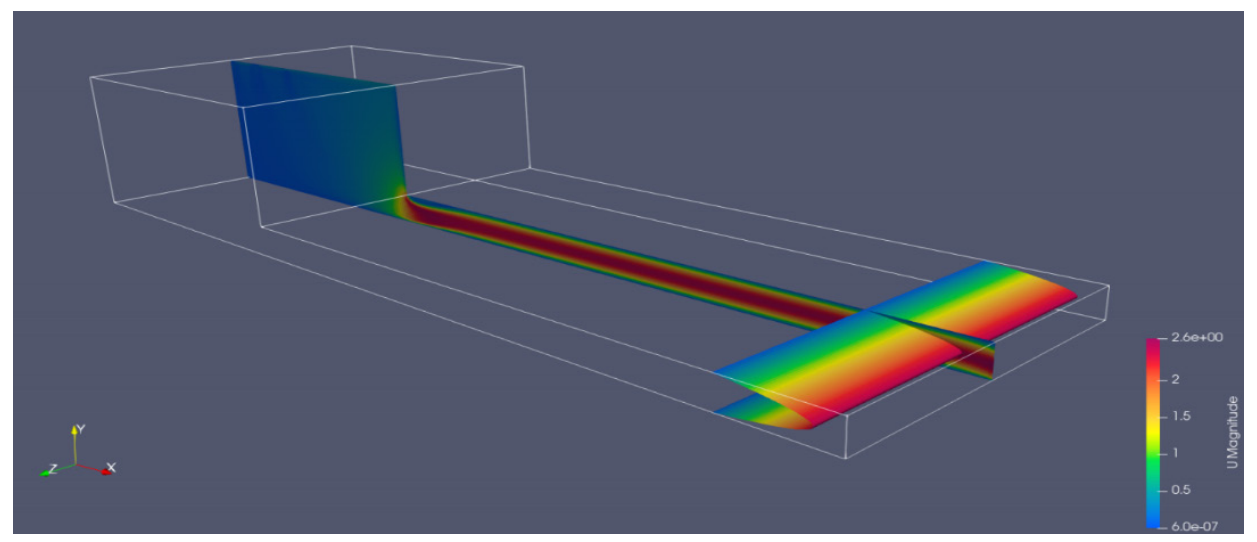

Fig. 4 Modelado parcial en ducto de estrada de pre-floculadores hacia floculadores.

\section{RESULTADOS}

Levantamiento preciso de prototipos en modelados 3D con características reales, los cuales proveen diferentes predicciones para los análisis cualitativos numéricos de ecuaciones diferenciales parciales (EDP); además el sistema con los cuales se crearon los modelos tridimensionales permite el análisis y modificación de cada una de las unidades que componen la planta para así realizar análisis de forma proporcional o en conjunto. [3]

La creación de nuestros modelos en AutoCAD nos permite la fácil cooperación con otros tipos de softwares de AUTODESK tal como es en el caso de AutoCAD civil 3D, pero también la importar nuestros modelos en otros tipos de archivos para ser empleados como puntos de entrada a otras herramientas de análisis diversos. Implementando de un entorno virtual altamente realista en CAD nos permite establecer una herramienta fuerte para futuros análisis y mejoramiento de la planta la cual podría incorporarse en el resto de las potabilizadoras y demás proyectos en el país.

\section{CONCLUSIONES}

Las aplicaciones que surgen con la modelación 3D para este proyecto son vastas y darán aperturas a una gran serie de estudios, análisis de mejoras e implementaciones con las cuales se verán recompensados tanto la comunidad científica, entidad estatal y las comunidades abastecidas por la planta potabilizadora de Chorrera.

Los modelos 3D en sistemas CAD permiten no solo crear un vistazo virtual a escala, si no que al poder incorporar propiedades que poseen los materiales de forma directas a una sección o pieza se crea una herramienta de análisis diversificada los cuales también permiten 
la exportación a otros formatos de los modelos para posteriormente ser empleados en otras plataformas o software que permitan unos análisis más exhaustivos.

\section{AGRADECIMIENTO}

A la Secretaría Nacional de Ciencia, Tecnología e Innovación (SENACYT) por el financiamiento del proyecto "Formulación de la optimización de una planta potabilizadora mediante modelación matemática y calibración en situ", adjudicado a la Universidad del Istmo bajo el contrato IOMA 19-006, del cual se desprende esta investigación. Además, al Instituto de Acueductos y Alcantarillados Nacionales (IDAAN) por el permanente apoyo brindado en el desarrollo de la investigación.

\section{Referencias}

[1] Y. Z. C. S. C. a. X. Z. S. Wang, Digital design for functionally graded material components rapid manufacturing, In Proceedings of GMP, 2008, pp. 491-497.

[2] K. K. a. P. T. V. Gupta, Reference based geometric modeling for heterogeneous objects, Computer Aided Design and Applications, vol. 9, 2012, pp. 155-165.

[3] A. C. \&. J. R. G. Rafael, Introducción al diseño paramétrico con Autodesk, 2005, pp. 305-355.

\section{Autorización y Licencia CC}

Los autores autorizan a APANAC XVIII a publicar el artículo en las actas de la conferencia en Acceso Abierto (Open Access) en diversos formatos digitales (PDF, HTML, EPUB) e integrarlos en diversas plataformas online como repositorios y bases de datos bajo la licencia CC:

Attribution-NonCommercial-ShareAlike 4.0 International (CC BY-NC-SA 4.0) https://creativecommons.org/ licenses/by-nc-sa/4.0/.

Ni APANAC XVIII ni los editores son responsables ni del contenido ni de las implicaciones de lo expresado en el artículo. 